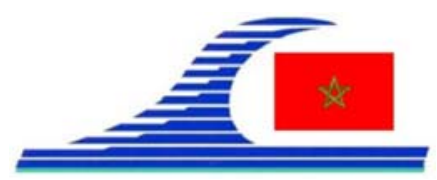

Conférence Méditerranéenne Côtière et Maritime

EDITION 2, TANGER, MAROC (2011)

Coastal and Maritime Mediterranean Conference

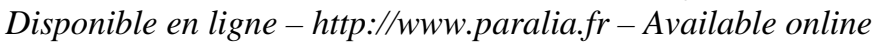

\title{
Dialogue sur les dynamiques côtières oranaises (Algérie) et picardes/normandes (France)
}

\section{Laurent DELABY ${ }^{1}$, Djilali BOURAS ${ }^{2}$, Mohammed KALLOUCHE ${ }^{2}$, Kais HUSSEIN BOUMEDIENNE ${ }^{2}$, Fouad ABDELGHANI ${ }^{2}$}

\author{
1. I.C. Ingénieurs Conseils, 143 Bd de Magenta, 75010 Paris, France. \\ l.delaby@yahoo.fr,http://www.ic-ingenieursconseils.fr/ \\ 2. Université d’Oran, Dép. Biologie, Fac. Sciences, Oran Es Sénia Algérie. \\ djilaloran@yahoo.fr
}

\section{Mots-clés :}

Aménagement du littoral - Développement économique

\section{Introduction}

Cet article est le fruit d'un travail débuté en 2007 lors du colloque international de Oran BEL01 (Biodiversité et Ecosystèmes Littoraux) . Ce travail de dialogue, d'échanges et de voyages entre partenaires algériens et français vise à la construction d'un dialogue sur le long terme afin de partager les enjeux touchant les littoraux connus et étudiés par les partenaires algériens, en particulier les côtes oranaises, et ceux touchant les littoraux connus et étudiés par les partenaires français, en particulier les côtes picardes et normandes, donnant ainsi un regard croisé sur les problématiques du développement et de l'adaptation à l'environnement (DELABY et al., 2009 ; BOURAS et al., 2009).

\section{Problématiques actuelles sur les côtes normandes et picardes, par L. Delaby}

Les côtes normandes et picardes sont actuellement le théâtre de la construction de projets d'une envergure considérable tels que :

- Centrales nucléaires EPR (Flamanville et Penly en projet) ;

- Parc Eoliens Off Shores (Le Tréport, Fécamp, Courseulles) ;

- Parc hydroliens.

Ces projets à classer dans la catégorie "grands travaux" sont d'une grande complexité et leur impact comporte un grand nombre d'aspects autant sociaux, économiques qu'environnementaux. Ils constituent une opportunité de développement pour la France au niveau économique en constituant "une vitrine" d'un savoir faire dans la construction de grands projets. La centrale EPR de Flammanville est par exemple un prototype, une tête de série, dont les promoteurs espèrent l'exportation dans les années à venir. Outre l'approvisionnement énergétique français, cet espoir justifie l'important investissement d'EDF de l'ordre de 6 milliards d'euros nécessaire à la construction de cette centrale. Les parcs éoliens offshores sont eux aussi de grands projets et constituent une innovation en France, ils consistent en l'implantation de plusieurs dizaines d'éoliennes 
La connaissance de la Mer :

un vecteur du développement durable en Méditerranée

hautes d'une centaine de mètres. Enfin, les projets de parcs hydroliens demandent encore un important effort de recherche développement avant de devenir opérationnels et de trouver leur place dans le panel énergétique français, les premières hydroliennes françaises commençant juste à être implantées en Bretagne près de l'île de Bréhat en septembre 2011.

Ces grands projets sont donc pleins de promesses et de défis, ils peuvent se révéler être de formidables moteurs de l'économie et de la connaissance en entrainant dans leur sillage une grande variété de métiers et de compétences, en suscitant des vocations, des emplois, et de l'activité économique.

Là est sans doute le plus grand défi de ces grands projets : ils sont pour la plupart portés par de grands opérateurs industriels. Leur échelle financière se situe au niveau du milliard d'euros. Par leur taille, ils vont avoir un impact écologique certain en modifiant les écosystèmes et l'environnement socio-économique des littoraux concernés. La question est donc de savoir comment concevoir et mettre en œuvre de manière harmonieuse ces projets afin qu'ils tirent dans leur périmètre d'influence toute l'économie vers le haut, sans engendrer de déséquilibres et de fractures dans la société. En d'autre terme, comment peuvent-ils susciter l'initiative, l'innovation, la création d'activité, un renforcement de la cohésion sociale ? Comment peuvent-ils s'intégrer dans leur environnement écologique de manière équilibrée afin qu'un apport d'un coté (économique par exemple) ne soit pas compensé par des effets délétères d'un autre (écologiques par exemple).

Tous ces aspects sont liés car si ces projets sont portés par de grands opérateurs, s'ils sont d'une échelle "macroscopique", l'ensemble de la société s'investit pour leur réalisation via le prix de l'électricité, et supportera les conséquences des choix mis en œuvre. L'ensemble des acteurs économiques et sociaux, notamment les PME, les jeunes entreprises, les associations, peut et doit apporter une contribution au perfectionnement de ces projets sur des échelles plus petites, dans le suivi environnemental, l'entretien de ces infrastructures, les formations, l'offre de services aux grands opérateurs, la diffusion des connaissances techniques et écologiques dans le grand public.

Cette nécessité "organique" de l'association complémentaire (symbiose) de la microéconomie à la macro-économie des littoraux réside dans l'analogie suivante : les PME, associations, petites structures sont en quelque sorte "les capillaires" qui irriguent localement le tissu économique français. Elles jouent le rôle d'un maillage du territoire, un rôle de relais, de création, d'expérimentation, elles ont leur utilité à leur échelle.

Il faut donc construire ensemble un dialogue soutenu des grands opérateurs qui portent ces grands projets avec les autres acteurs économiques et sociaux, et mettre en place des mécanismes de synergies, de soutiens mutuels et de partenariats. 


\section{Problématique actuelle sur les littoraux oranais. Identification et stabilisation des formes côtières sur les littoraux oranais, par D. Bouras, K. Hussein, S. Mouffok, F. Abdelghani}

Une zone côtière est communément liée à une appellation de lieu, généralement en rapport avec la portion continentale du paysage, octroyé par les différents usagers. Cela montre l'importance des observations des groupes sociaux, les dénominations des lieux expliquent souvent leurs caractéristiques. Ces attributions peuvent être des noms historiques (Montagne des lions), des noms de tribus, d'une ville ou village, par exemple, la falaise de Kristel (Est d'Oran), Maddagh (entre Oran et Ain Temouchent), ou selon la forme caractérisant le paysage (par exemple Pointe ou Cap de l'aiguille, Arzew). Parfois, en fonction de leur fréquentation (Plage des mouches, Ain Témouchent). Ou encore la nature singulière du site côtier (Plage Sablette).
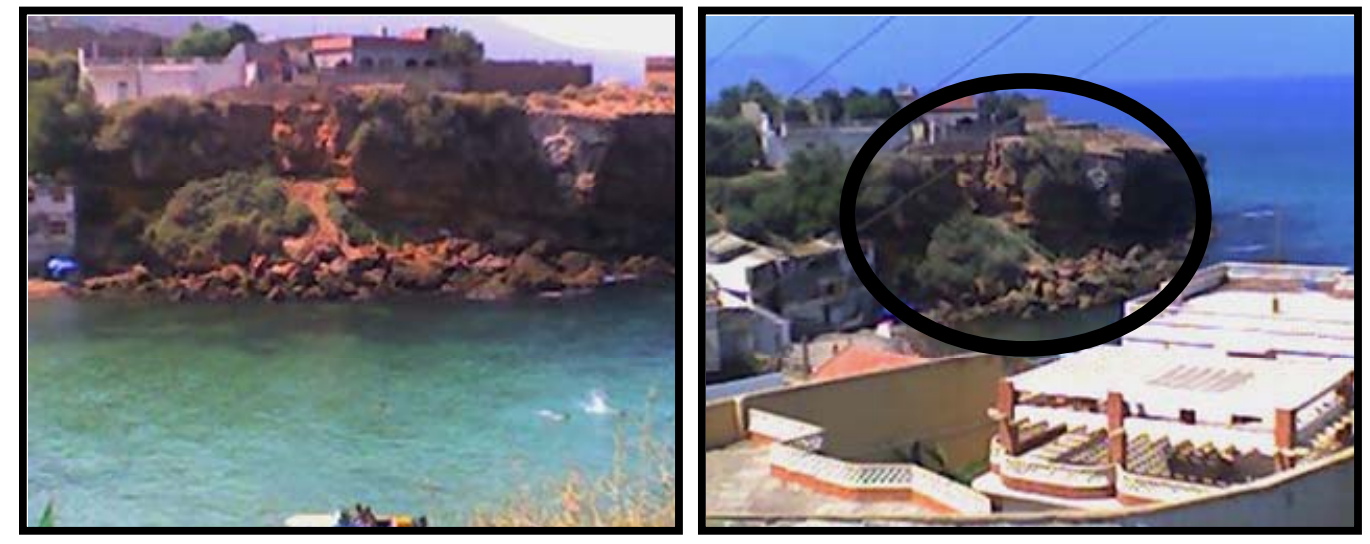

Figure 2. Effondrement de la falaise menaçant de nombreuses installations touristiques le long de la côte de Kristel.

Depuis son occupation des paysages côtiers, les hommes combattent contre le recul des côtes avec différents moyens de défense, de reconfiguration et d'adaptation (épis, murs, enrochements pour la protection des habitations, des routes ou encore un champ cultivé...). Le recul du trait de côte se traduit par une régression du continent et des pertes aux biens de l'homme (figure 2) et une modification de la faune et de la flore. L’impact des actions prises doit être bien étudié afin de prévenir toute sorte de complications comme l'incidence de changements sur l'amont qui influencent l'aval du transit littoral. L’érosion dunaire des côtes peut être amplifiée par l'action anthropique (exemple : circulation de véhicules, vol de sables, construction, route, désenclavement de sentiers,...). L’organisation paysagère oranaise a largement été modifiée en raison des aménagements effectués (Macta, Kristel, Ain El Turk, ports pétroliers de Arzew...) qui ont affaibli voire brisé l'apport en alluvions du transit littoral et des différents cours d'eaux, en particulier au niveau de la région de la Macta. Par ailleurs, les besoins augmentés en sable et en gravier pour les nombreux projets d'aménagements de 
La connaissance de la Mer :

un vecteur du développement durable en Méditerranée

l'industrie et les différentes constructions entraînent une surexploitation des réserves des lits des cours d'eau et celle du domaine côtier (cas de Terga, Ain Témouchent, Arzew). De nombreux cours d'eaux ont été déviés de leur trajectoire initiale (Macta, Kristel, Oued El Halouf), provoquant une déstabilisation de l'alimentation des eaux côtières. Signalons l'intensité des travaux de construction sur des espaces non constructibles, engendrant un fort danger sur l'homme et une fragilisation et dégradation des reliefs et de l'écosystème. Ces aménagements perturbent les conditions hydrodynamiques et favorisent une forte érosion actuelle.

La question relative aux paysages côtiers est à replacer dans un rapport plus général sur le devenir des littoraux magrébins. L'identification des facteurs de déséquilibre est très importante dans l'évolution du trait de côte.

\section{Conclusion - synthèse}

Ce dialogue franco-algérien permet avec humilité de mettre en perspective les problématiques touchant les littoraux en comparant les enjeux, les approches, la nature de la relation de l'Homme avec son environnement. Les mots pour les décrire de part et d'autres de la Méditerranée ne sont pas forcément identiques, les méthodes de raisonnement non plus, mais la volonté de recherche des solutions optimales identique. En France, la complexité des questions d'approvisionnement énergétique semble occuper les réflexions actuelles, sans doute temporairement devant les problèmes d'érosion et de protection du littoral, avec la construction de grandes unités de production énergétiques. Il y a encore de grands défis pour associer à ces projets sur les littoraux les acteurs majeurs que sont les PME et les jeunes entreprises. En Algérie, une réflexion sur les aménagements conséquents au développement économique tels que les routes, les prélèvements de sédiments pour la construction, les complexes touristiques, les grandes infrastructures portuaires liées à l'industrie pétrolière cherche à comprendre et à construire un rapport équilibré de ce développement avec son cadre, la Nature.

\section{Références bibliographiques}

BOURAS D., DELABY L., HUSSEIN K., MOUFFOK S., ABDELGHANI F. (2009). Espace Littoral et Dynamique Paysagère (Littoral Oranais, Algérie). Bulletin des Sciences Géographiques n² 24, $2^{\text {ème }}$ semestre 2009.

DELABY L., BOURAS D., MOUFFOK S. (2009). Introduction à une étude comparée du fonctionnement de la dynamique du trait de côte du littoral algérien occidental et des côtes picardes et normandes françaises : mécanismes et enjeux. Conférence Méditerranéenne Côtière et Maritime, Edition 1, Hammamet, Tunisie, pp 187-190. doi:10.5150/cmcm.2009.047-3 